\title{
Depletion of Neutrophils Protects Against L-Arginine-Induced Acute Pancreatitis in Mice
}

\author{
Guolin Chen Feng Xu Jing Li Shiqi Lu \\ Emergency Department, The First Affiliated Hospital of Soochow University, Suzhou, China
}

\section{Key Words}

Acute pancreatitis $\cdot$ Neutrophils $\cdot N F-\kappa B$

\begin{abstract}
Background/Aims: Acute pancreatitis (AP) is an inflammatory disease characterized by acinar cell damage and inflammation of the pancreas with infiltration of leukocytes, predominantly neutrophils. We investigated whether neutrophil depletion protects against experimental AP induced by L-arginine. Methods: AP was induced in C57BL/6 mice via two intraperitoneal $\mathrm{L}$-arginine $(4 \mathrm{~g} / \mathrm{kg})$ injections. Mice were pretreated with 250 and $100 \mu \mathrm{g}$ anti-Gr-1 antibody via intraperitoneal injection at 24 and $4 \mathrm{~h}$, respectively, before L-arginine challenge for neutrophil depletion. At 48 and $72 \mathrm{~h}$ after injection, the severity of AP was determined with the aid of biochemical and histological analyses. Amylase and MPO activity was detected using specific assay kits. The plasma cytokines levels were detected using ELISA. Results: Neutrophil depletion resulted in significantly reduced plasma amylase levels in pancreas, myeloperoxidase (MPO) activity in pancreas and lung, reactive oxygen species (ROS) generation and cell apoptosis, and decreased circulating neutrophil, tissue damage as well as expression levels of nuclear factor NF-KB. Conclusion: Neutrophil depletion is capable of reducing tissue damage of pancreas and lung in mice with acute pancreatitis.
\end{abstract}

Copyright (C) 2015 S. Karger AG, Basel

\section{Introduction}

Acute pancreatitis (AP) is an inflammatory disease of the pancreas. Diagnosis of AP is based on increased concentrations of amylase and lipase in serum, along with acute abdominal pain $[1,2]$. Clinically, most patients with AP display mild pancreatic injury without complications after therapy, while about $20 \%$ have a life-threatening disease with

Shiqi Lu

KARGER 125
Emergency Department, The First Affiliated Hospital of Soochow University,

No. 188, Shizi Road, Suzhou 215006 (China)

Fax+860512 67780296, E-Mail lushiqi1212@163.com 


\section{Cellular Physiology Cell Physiol Biochem 2015;35:2111-2120 \begin{tabular}{c|c|c|}
\hline DOI: 10.1159/000374017 & O 2015 S. Karger AG, Basel
\end{tabular} \begin{tabular}{l|l|l|l|l|l} 
and Biochemistry Published online: April 07, 2015 & www.karger.com/cpb \\
\hline
\end{tabular} \\ Chen/Xu/Li/Lu: Role of Neutrophil Depletion in AP Protection}

local and systemic complications and experience multiple organ dysfunction [3, 4]. To date, intense efforts have been made to uncover the exact mechanisms regulating the initiation and severity of AP. Earlier studies showed that the severity of AP can be determined based on pathophysiological components within pancreatic acinar cells, such as activation of trypsinogen and nuclear factor (NF)- $\kappa B$, release of pro-inflammatory cytokines, recruitment of neutrophils and apoptosis of acinar cells [5-8]. In this light, the inflammatory response plays crucial roles in AP, and effective therapy should focus on key components of the immune system, such as neutrophils.

Several studies have reported that upregulated activity of myeloperoxidase (MP0), a biochemical marker of neutrophil infiltration, is associated with disease development in sodium taurocholate-induced, cerulein-induced, and L-arginine-induced AP in both mice and rats $[4,5,9-12]$. Moreover, neutrophil depletion induced by pretreatment of mice with antineutrophil serum reduces the severity of pancreatitis and prevents pancreatitis-associated lung injury $[13,14]$. An earlier study by Pandol and colleagues demonstrated that the activity of neutrophils infiltrating the pancreas in cerulean-induced AP is mediated through NADPH oxidase products [15].

In the present study, L-arginine-induced AP in mice was characterized by increased pancreatic injury, plasma amylase and MPO activities, and higher frequency of neutrophils in the pancreas. Depletion of neutrophils via intraperitoneal injection with an anti-Gr-1 antibody (RB6-8C5) [16] resulted in significant protection against L-arginine-induced pancreatic inflammation, as evident from the marked reduction in plasma amylase in pancreas and MPO activity in pancreas and lung, suppression of the reactive oxygen species (ROS) content, and decreased apoptosis of pancreatic cells. These effects of neutrophil depletion on AP were associated with decreased NF- $\kappa$ B expression. Our findings collectively indicate a critical role of neutrophils in the development of AP.

\section{Materials and Methods}

\section{Experimental procedures}

All animal related procedures were approved by the Animal Research Committee at Soochow University. Animal experiments were performed in accordance with the established International Guiding Principles for Animal Research. C57BL/6 mice (male, 20-25 g) were maintained under a $12 \mathrm{~h} \mathrm{light/dark}$ cycle at $21-24^{\circ} \mathrm{C}$ in the Animal Housing Unit. Animals were allowed to acclimatize for at least 1 week before experimental procedures were undertaken, and randomly assigned to control or experimental groups.

\section{Induction of AP and neutrophil depletion}

L-Arginine hydrochloride was purchased from Sigma Chemical (St. Louis, MO). A sterile solution of L-arginine hydrochloride (8\%) was prepared in normal saline (pH 7.0). Mice were randomly assigned to four groups. In the control group, animals were injected twice intraperitoneally using normal saline $1 \mathrm{~h}$ apart. In the AP group, animals were injected intraperitoneally with L-arginine solution at two doses of $4 \mathrm{~g} / \mathrm{kg}$ body weight each $1 \mathrm{~h}$ apart [17]. After treatment, animals were returned to their cages and allowed free access to food and water. In the AP+aGr-1 group, to deplete neutrophils, animals were injected intraperitoneally with 250 and $100 \mu \mathrm{g}$ anti-Gr-1 monoclonal antibody (mAb) per mouse (RB6-8C5, BD Biosciences, San Diego, CA) at 24 and $4 \mathrm{~h}$ before the L-arginine challenge, respectively [16]. In the AP+IgG2b Group, control rat IgG2b (BD Biosciences) for anti-Gr-1 treatment was injected at equivalent doses. Animals were killed at specific time-points via $\mathrm{CO}_{2}$ asphyxia and blood samples collected to determine serum amylase levels. Pancreas samples from mice were rapidly collected and fixed in $4 \%$ paraformaldehyde for hematoxylin and eosin (H\&E) staining. Portions of organs were stored at $-80^{\circ} \mathrm{C}$ for further investigation.

\section{Histological scores}

Pancreatic damage was evaluated using a scoring system ranging from 0 (absent) to 4 (extensive) as previously described [18]. Total histological scores represent the sum of scores for inflammatory cell infiltration, edema, acinar cell necrosis and hemorrhage. Lung injury was assessed according to pulmonary 


\section{Cellular Physiology Cell Physiol Biochem 2015;35:2111-2120 \begin{tabular}{l|l} 
DOI: 10.1159/000374017 & (C) 2015 S. Karger AG, Basel
\end{tabular} and BiOChemistry Published online: April 07, $2015 \quad$ www.karger.com/cpb \\ Chen/Xu/Li/Lu: Role of Neutrophil Depletion in AP Protection}

edema, inflammatory changes, alveoli and interstitial tissue hemorrhage. Pathological features were scored on a scale from 0 (normal) to 3 (severe) in terms of changes.

\section{Amylase estimation}

A kinetic spectrophotometric assay was used to measure plasma amylase activity, as described previously [19]. Plasma samples were incubated with amylase reagent for $2 \mathrm{~min}$ at $37^{\circ} \mathrm{C}$, and absorbance measured at $405 \mathrm{~nm}$ according to the manufacturer's protocol. The changes in absorbance were used to estimate amylase activity.

\section{MPO estimation}

Fresh tissue samples were homogenized immediately on ice in 5 volumes of normal saline. The MPO assay kit (Nanjing Jiancheng Corp., Nanjing, China) was used to measure MPO activity according the manufacturer's recommendations. One unit of MPO activity was defined as that required to degrade $1 \mathrm{mmol}$ hydrogen peroxide at $37^{\circ} \mathrm{C}$. MPO activity was expressed as units per milligram $(\mathrm{U} / \mathrm{mg})$.

\section{Reverse transcription and quantitative real-time PCR}

Total RNA was extracted from tissue using TRIzol reagent (Invitrogen, Carlsbad, CA), according to the manufacturer's instructions. The PrimeScript RT Reagent Kit (TaKaRa, Dalian, China) was used to synthesize complementary DNA from 500 ng total RNA. Quantitative real-time PCR was performed with SYBR Green PCR master mix (Applied Biosystems, Foster City, CA). NF- $\mathrm{BB}$ p65 expression was determined using the primers 5'-ATACCACCAAGACCCACCCC-3' (forward), 5'-TGAGGAGGGTCCTTGGTGAC-3' (reverse). The relative expression of p65 was measured with the $2^{-\Delta C \mathrm{CT}}$ method using $\beta$-actin mRNA for normalization.

\section{Western blot analysis}

Pancreatic tissues were homogenized in RIPA lysis buffer, along with protease inhibitor (SigmaAldrich), and electrophoresed on a 10\% SDS gel. Proteins were transferred to polyvinylidene fluoride membrane, followed by blocking with $5 \%$ skimmed milk. Blots were subsequently incubated with primary antibodies against cleaved caspase 3, p65 and GAPDH (Abcam, Cambridge, UK) at $4^{\circ} \mathrm{C}$ overnight. After further incubation with horseradish peroxidase (HRP)-conjugated secondary antibodies (Cell Signaling Technology, Beverly, MA), proteins were detected using enhanced chemiluminescence (Pierce, Rockford, IL).

\section{Measurement of ROS}

ROS production was detected using CM-H2DCFDA (Molecular Probes, Carlsbad, CA), a cell-permeable dye that remains non-fluorescent until acetate groups are removed [20]. In brief, $200 \mu \mathrm{l}$ cell suspensions $\left(10^{5} \mathrm{cells} / \mathrm{ml}\right)$ were seeded into a 96 -well plate in the presence of CM-H2DCFDA for $45 \mathrm{~min}$ at $37^{\circ} \mathrm{C}$. Analysis of signal intensity was carried out using a fluorescent plate reader and ROS production calculated based on a $\mathrm{H}_{2} \mathrm{O}_{2}$ standard curve.

\section{TUNEL assay}

Apoptosis was determined using TUNEL, a method for detecting DNA fragmentation. Pancreatic tissues were fixed by immersion in $10 \%$ buffered formaldehyde phosphate for at least one day, dehydrated and embedded in paraffin wax. The TUNEL assay was performed on $5-\mu \mathrm{m}$ thick sections using a cell apoptosis detection kit (Promega, Madison, WI), according to the manufacturer's protocol.

\section{Flow cytometric analysis}

Pancreatic cell suspension was prepared as described previously [21]. Briefly, pancreatic tissues were sheared gently and digested with collagenase $\mathrm{V}(1 \mathrm{mg} / \mathrm{ml})$ for $15 \mathrm{~min}$, followed by passage through a $40 \mu \mathrm{m}$ filter. The cell suspension was centrifuged at $1500 \mathrm{rpm}$ for $10 \mathrm{~min}$, and subsequently washed in phosphate buffered saline. Cells were incubated for 10 min with a Fc $\gamma$ receptor blocker, CD16/32 (BD Biosciences). Aliquots of cells $\left(10^{6}\right)$ were stained with fluorescein isothiocyanate (FITC)-conjugated anti-CD3, anti-CD11b, anti-F4/80, and phycoerythrin (PE)-conjugated anti-NK1.1 and anti-Gr-1 antibodies (BD Biosciences). Peripheral blood mononuclear cells harvested from eyeballs of mice after density gradient centrifugation were incubated with fluorescein isothiocyanate (FITC)-conjugated anti-CD11b and phycoerythrin (PE)conjugated anti-Gr-1 antibodies to detect circulating neutrophils. Flow cytometric analysis was carried out 
A
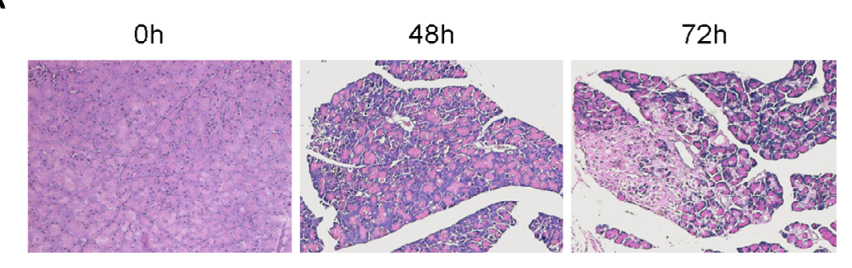

B

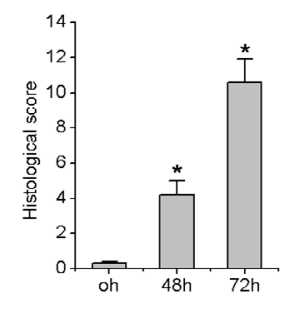

C

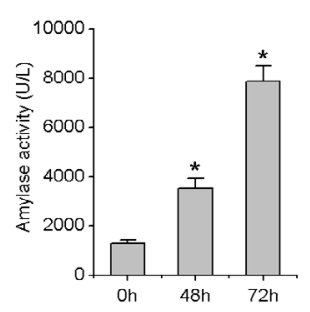

D

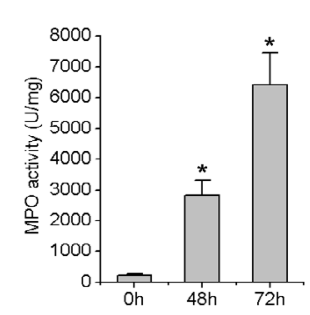

$\mathrm{F}$

$E$
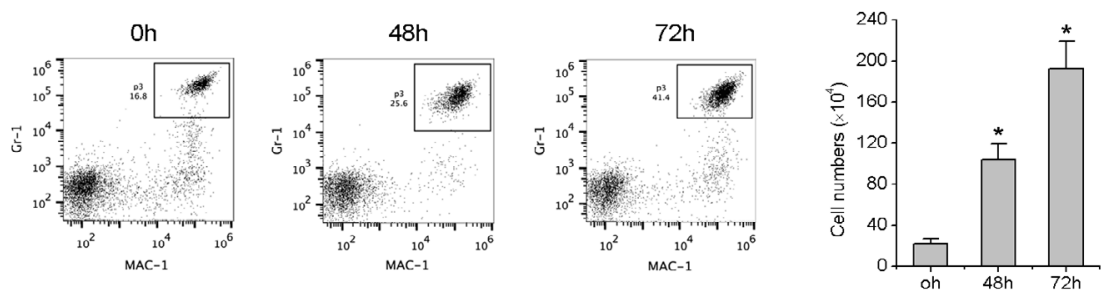

Fig. 1. Neutrophil infiltration in L-arginine-induced AP mice. (A) Histopathological changes in mouse pancreas after L-arginine administration at different time-points. Representative H\&E-stained micrographs are presented $(\times 20)$. (B) Histological scores. (C) Plasma amylase activity. (D) Pancreatic myeloperoxidase (MPO) activity. (E) Representative flow cytometric analysis and absolute number of neutrophils from pancreas at the indicated time points following administration of L-arginine. (F) Absolute numbers of CD3 T cells, NK cells, NKT cells and macrophages per pancreas at the indicated time-points following administration of L-arginine. Data are presented as means \pm SD $\left(n=5\right.$ per group). ${ }^{*} P<0.05$ versus 0 h group.

on a FACSCalibur (BD Biosciences) to calculate the quantity of each cell type (T cells, NK cells, NKT cells, neutrophils and macrophages).

\section{Statistical analysis}

All data were calculated as means \pm SD are from at least three separate experiments. Statistical analysis was performed by applying the unpaired two-tailed Student's $t$-test, with significance assigned to $P<0.05$ in all cases.

\section{Results}

Increased infiltration of neutrophils in L-arginine-induced pancreatitis of mice

The successful development of a novel mouse model of acute pancreatitis induced by L-arginine has been previously reported [17]. Evaluation of histology of the pancreas in the L-arginine-administered group using hematoxylin and eosin-stain (HE) revealed disruption of normal histoarchitecture, distended interlobular spaces, acinar cell vacuolization and neutrophil infiltration over time after induction of acute pancreatitis (Fig. 1A and B). Plasma amylase levels were significantly increased in the AP group at 48 and $72 \mathrm{~h}$ after L-arginine administration, compared with the control group (Fig. 1C). Furthermore, mice of AP group 


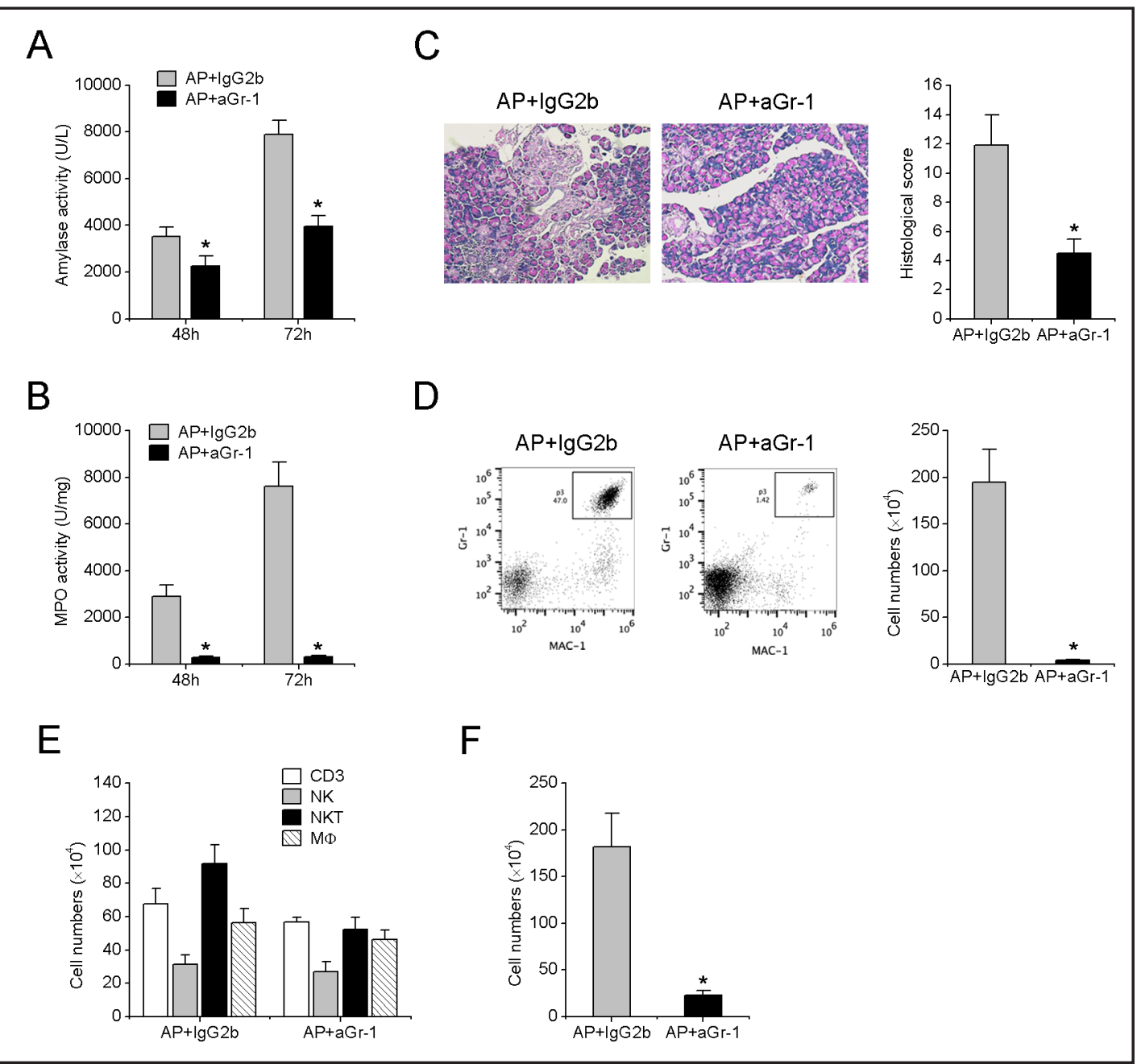

Fig. 2. Neutrophil depletion inhibits tissue damage and innate immune cell accumulation. Normal mice were injected with L-arginine ( $4 \mathrm{~g} / \mathrm{kg}$ ) intraperitoneally on day 0.250 and $100 \mu \mathrm{g}$ anti-Gr-1 (RB6-8C5) mAb was injected intraperitoneally at 24 and $4 \mathrm{~h}$, respectively, prior to the L-arginine challenge. IgG2b was injected at equivalent doses in the AP+IgG2b group. (A) Plasma amylase activity. (B) MPO activity. (C) Histological analysis of pancreatic sections with hematoxylin-eosin stain and histological score. (D) Representative flow cytometric analysis and quantity of neutrophils $72 \mathrm{~h}$ after IgG2b (AP+IgG2b) and anti-Gr-1(AP+aGr-1) treatment. (E) Absolute numbers of CD3 T cells, NK cells, NKT cells and macrophages per pancreas in AP+IgG2b and AP+aGr-1 group. (F) Absolute number of neutrophil in peripheral blood mononuclear cells. Data are presented as means $\pm \mathrm{SD}\left(\mathrm{n}=5\right.$ per group). ${ }^{*} P<0.05$ versus $\mathrm{AP}+\mathrm{IgG} 2 \mathrm{~b}$ group.

displayed markedly increased pancreatic MPO activity at $48 \mathrm{~h}$ and $72 \mathrm{~h}$ after administration of L-arginine compared with the control group (Fig. 1D), consistent with the patterns of plasma amylase.

As neutrophil infiltration to the pancreas was observed in L-arginine-induced AP, we determined the number of neutrophils $\left(\mathrm{Gr}-1^{+} \mathrm{Mac}-1^{+}\right)$and other leukocytes, such as $\mathrm{T}$ cells $\left(\mathrm{CD}^{+} \mathrm{NK} 1.1^{-}\right)$, NK cells $\left(\mathrm{NK} 1.1^{+} \mathrm{CD}^{-}\right)$, NKT cells $\left(\mathrm{NK} 1.1^{+} \mathrm{CD}^{+}\right)$and macrophages $\left(\mathrm{F} 4 / 80^{+}\right)$, based on two-parameter flow cytometric analysis. As shown in Fig.1E, the quantity of neutrophil significantly increased in L-arginine-induced AP mice, compared with the control group, while the number of CD3 T cells, NK cells, NKT cells and macrophages was increased to a moderate extent after L-arginine administration (Fig.1F), indicating a crucial role of neutrophil in the development of AP. 
Fig. 3. Neutrophils depletion leads to decreased lung tissue damage. Normal mice were injected with L-arginine $(4 \mathrm{~g} / \mathrm{kg})$ intraperitoneally on day 0 . In anti-Gr-1 treated mice, 250 and $100 \mu \mathrm{g}$ anti-Gr-1 (RB6-8C5) $\mathrm{mAb}$ were injected intraperitoneally 24 and $4 \mathrm{~h}$ lrespectively before L-arginine challenge. IgG2b was injected at an equivalent dose. (A) MPO activity. (B) Histological analysis of lung sections with hematoxylin-eosin stain. (C) Histological scores. Data are presented as means \pm SD ( $n=5$ per group). ${ }^{*} P<0.05$ versus control group, ${ }^{* *} P<0.05$ versus AP group.

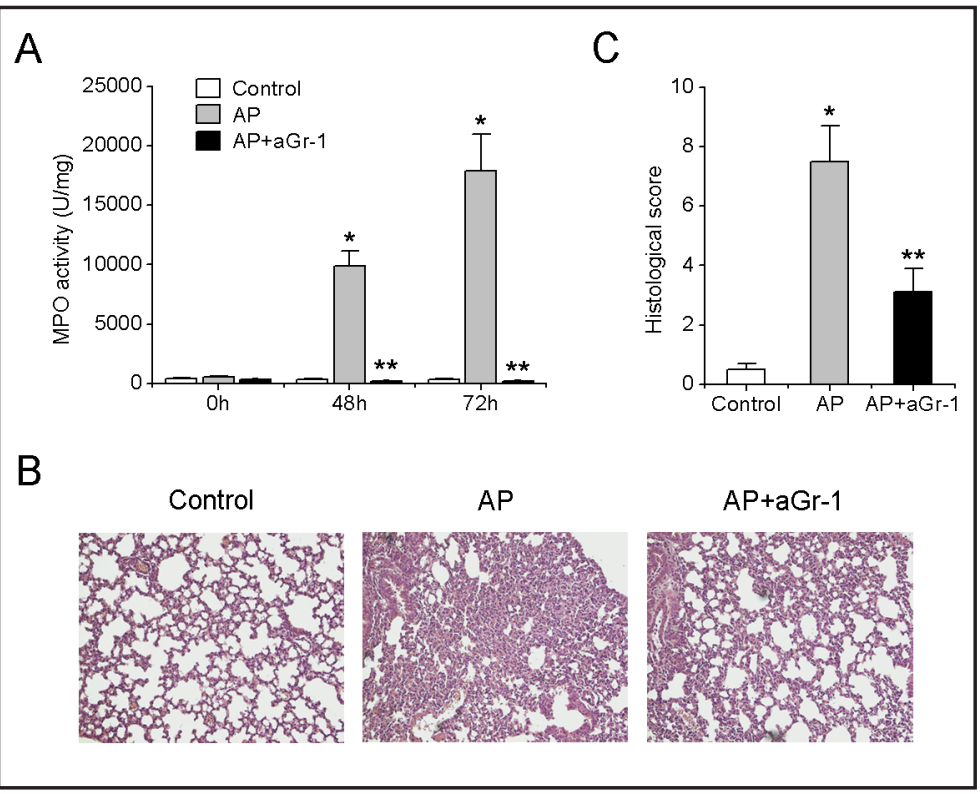

Depletion of neutrophils decreases pancreatic injury and innate immune cell accumulation in L-arginine-induced mice

To determine whether infiltrated neutrophils play a role in the development of L-arginine-induced AP, neutrophils were depleted via intraperitoneal injection of anti-Gr-1 $\mathrm{mAb}$ at $24 \mathrm{~h}(250 \mu \mathrm{g}$ per mouse $)$ and $4 \mathrm{~h}(100 \mu \mathrm{g}$ per mouse $)$ before L-arginine administration ( $4 \mathrm{~g} / \mathrm{kg}$ intraperitoneally). As shown in Fig. $2 \mathrm{~A}$ and $\mathrm{B}$, anti-Gr-1 treatment led to a significant decrease in plasma amylase and MPO activities, compared with those of IgG2b-treated control mice. Furthermore, histological analysis of pancreas sections revealed that the area of tissue damage is considerably reduced in neutrophil-depleted AP mice (AP+aGr-1), compared with IgG2b mAb-treated mice (AP+IgG2b) in which L-arginine caused severe tissue damage (Fig. 2C). Our findings demonstrate that depletion of neutrophils is an effective means to decrease pancreatic injury in an L-arginine-induced AP mouse model.

We additionally examined the effects of neutrophil depletion on leukocyte accumulation in pancreas. Leukocytes were isolated from mouse pancreas $72 \mathrm{~h}$ after L-arginine treatment. The frequrecy and number of neutrophils were significantly decreased in mice treated with anti-Gr-1 mAb (AP+aGr-1), compared to those treated with IgG2b mAb (AP+IgG2b) (Fig. 2D). However, anti-aGr-1 had a weak inhibitory effect on infiltration of other innate immune cells in the pancreas (Fig. 2D). Furthermore, circulating neutrophils from peripheral blood mononuclear cells were reduced by about $90 \%$ in the AP+aGr-1 group, compared with the AP+IgG2b group (Fig. 2F).

\section{Effect of neutrophil depletion on lung MPO and histology in L-Arginine-induced AP}

The effects of neutrophil depletion on lung injury were additionally analyzed. As shown in Fig. 3A, MPO activity in lung was significantly increased in AP group at 48 and $72 \mathrm{~h}$ after L-arginine administration, compared with control group, which was markedly decreased upon neutrophil depletion. Hematoxylin and eosin staining (HE) was performed for detection of lung injury in mice with L-Arginine-induced AP. As shown in Fig. 3B and C, pulmonary edema, alveolar thickening and neutrophils infiltration were more severe in the AP group, compared with control group. Lung tissue damage was significantly attenuated upon neutrophil depletion at the $72 \mathrm{~h}$ time-point.

Effect of neutrophil depletion on plasma cytokines level in L-Arginine-induced AP

Inflammatory reaction is reported to be associated with AP and a number ofcytokines, such as tumor necrosis factor $\alpha$ (TNF- $\alpha$ ), IL- 6 and IL-10 play regulatory roles in AP [22]. 
Fig. 4. Neutrophils depletion leads to decreased plasma levels of TNF- $\alpha$, IL- 6 and IL-10. Normal mice were injected with L-arginine $(4 \mathrm{~g} / \mathrm{kg})$ intraperitoneally on day 0 . In anti-Gr-1 treated mice, 250 and $100 \mu \mathrm{g}$ anti-Gr-1 (RB68C5) $\mathrm{mAb}$ were injected intraperitoneally 24 and $4 \mathrm{~h}$, respectively, before L-arginine challenge. IgG2b was injected at an equivalent dose. Plasma levels of TNF- $\alpha$ (A), IL-6 (B) and IL-10 (C)was measured by ELISA. Data are presented as means \pm SD $(\mathrm{n}=5$ per group). ${ }^{*} P<0.05$ versus control group, ${ }^{* *} P<0.05$ versus AP group.

Fig. 5. Neutrophil depletion decreases ROS generation and apoptosis. (A) ROS production by pancreatic acinar cells of control, AP and $\mathrm{AP}+\mathrm{aGr}-1$ mice. (B) Western blot detection of cleaved caspase 3 protein expression. (C) TUNEL detection of cellular apoptosis. Data are presented as means \pm SD. ${ }^{*} P<0.05$ versus control group, ${ }^{* *} P<0.05$ versus AP group.
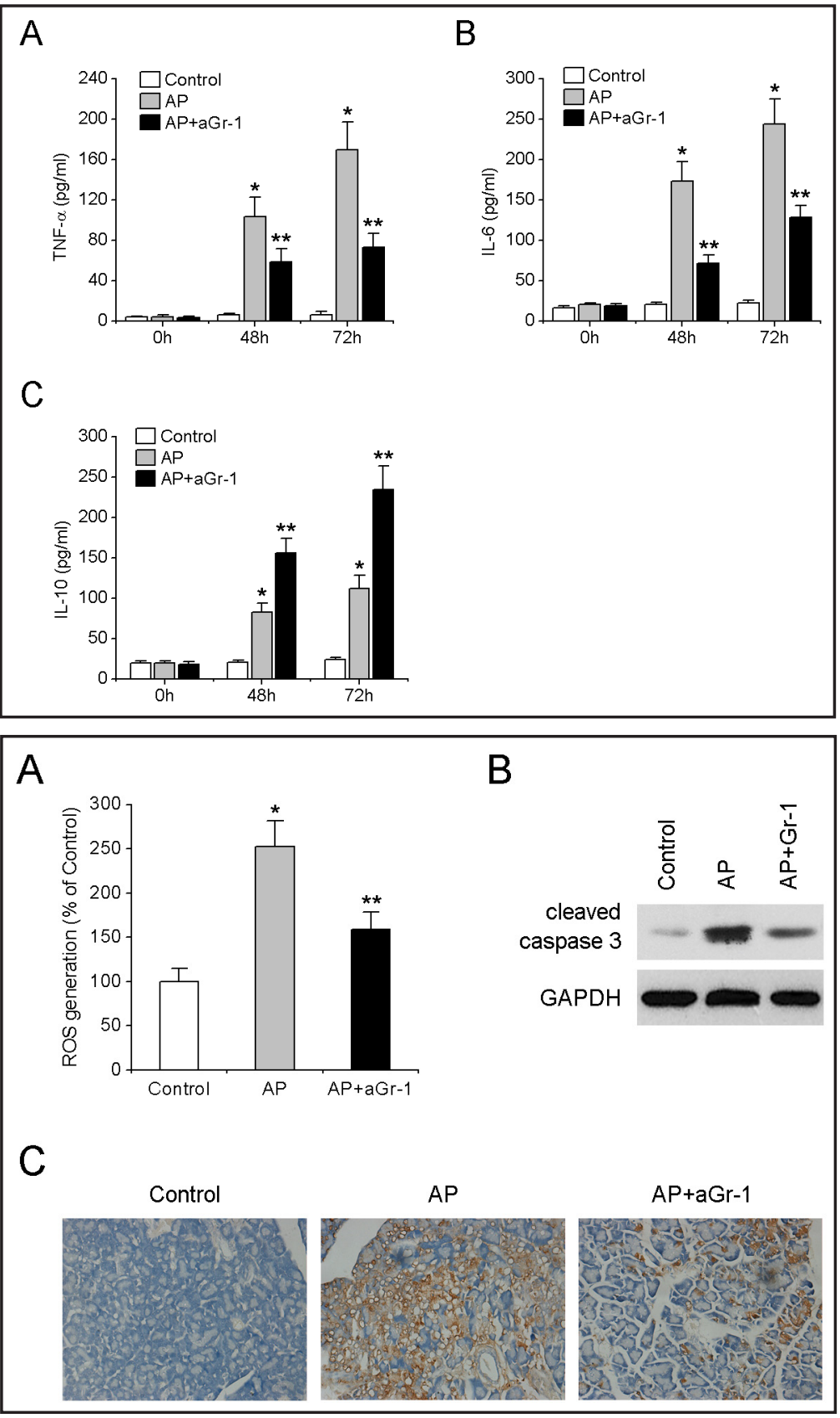

Accordingly, plasma levels of these cytokines were determined using ELISA. As shown in Fig. 4, levels of all the cytokines examined were significantly higher in the AP group than the control group. Notably, neutrophil depletion led to decreased plasma levels of TNF- $\alpha$ and IL6, but increased plasma IL-10, compared with the AP group.

Effects of neutrophil depletion on L-arginine-induced apoptosis in pancreas

Previous studies have reported the ability of neutrophils to produce and release a variety of cytotoxic agents, such as reactive oxygen and nitrogen species, which cause apoptosis [23]. As shown in Fig. 5A, reactive oxygen species (ROS) generation was markedly increased in leukocytes isolated from L-arginine-induced AP mice, compared with control mice. However, upon depletion of neutrophils with anti-Gr-1 treatment (AP+aGr-1), we observed significant reduction of ROS generated from leukocytes, clearly indicating an important role of neutrophils in ROS generation. Expression of cleaved caspase-3, a commonly used marker 
Fig. 6. Neutrophil depletion inhibits nuclear translocation of NF$\kappa \mathrm{B}$. Detection of pancreatic tissue expression of NF- $\mathrm{KB}$ p65 protein in the nucleus using real-time PCR and western blot $(\mathrm{A}, \mathrm{B}) .{ }^{*} P<0.05$.

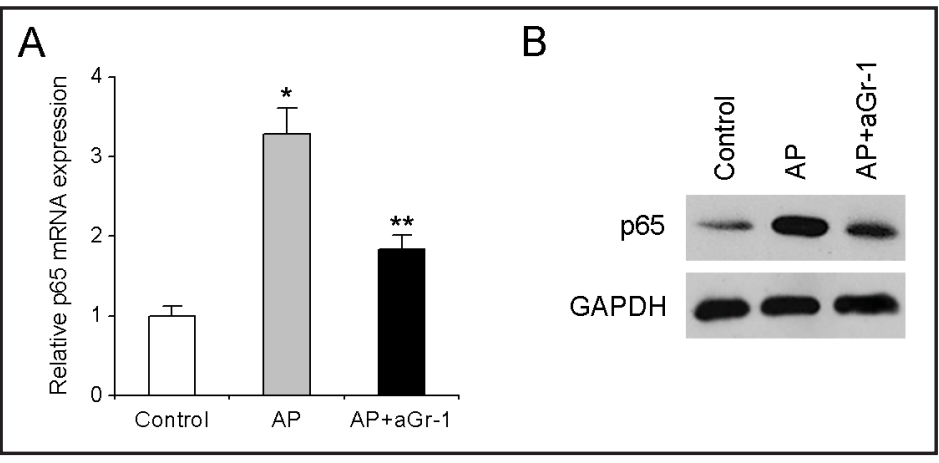

of cell apoptosis, was detected using western blot analysis. Our results disclosed markedly higher caspase-3 expression in AP mice, compared with that in control mice, which was significantly reduced under conditions of neutrophil depletion (Fig. 5B). Apoptosis levels were additionally determined using transferase-mediated deoxyuridine triphosphatebiotin nick-end labeling (TUNEL). As shown in Fig. 5C, cellular apoptosis in AP mice was significantly increased, compared with that in control mice, but decreased markedly upon anti-aGr-1 treatment. These results collectively suggest that neutrophil depletion results in reduction of ROS generation and cell apoptosis and exerts a protective role in pancreatic acinar cells during AP.

\section{Effects of neutrophil depletion on NF- $\kappa B$ expression in pancreas}

Given that the NF- $\kappa B$ pathway participates in the pathogenesis and development of AP [6], we examined the mRNA and protein expression levels of NF- $\kappa B$ p65 via real-time PCR and western blot analysis to determine the effects of neutrophil depletion on NF- $\kappa B$ in pancreas. As shown in Fig. 6A, p65 mRNA expression was significantly downregulated upon neutrophil depletion (AP+aGr-1), compared with that in non-treated AP mice. The patterns of NF- $\kappa B$ p65 protein expression were consistent with those of mRNA expression (Fig. 6B).

\section{Discussion}

Acute pancreatitis (AP) is a life-threatening pancreatic disease with significant morbidity and high mortality, but no specific therapies are currently available $[24,25]$. The development of AP is a multistep process characterized by inflammation, edema and vacuolization [24]. Clarification of the exact mechanism regulating the pathogenesis and development of AP is essential for effective therapy, which remains an unmet medical need. Infiltration of neutrophils, an essential component of the host innate immunity system, is a well-known hallmark of tissue injury in AP and contributes to the pathogenesis and development of the disease [26]. In the present study, we showed that depletion of neutrophils in an L-arginineinduced AP mouse model protects against AP, as evident from downregulation of plasma amylase and MPO activity and reduction in pancreatic tissue damage. Lung tissue damage and plama levels of inflammatory cytokines were decreased following neutrophil depletion. ROS generation and cleaved caspase 3 protein expression in pancreas were decreased significantly upon complete neutrophil depletion via anti-aGr-1 treatment. Apoptosis levels detected using TUNEL were markedly reduced under conditions of neutrophil depletion, consistent with the changes in caspase 3 expression, indicating that the protective role of neutrophil depletion against AP is achieved through suppression of cellular apoptosis in the pancreas. Furthermore, the NF- $\kappa B$ pathway is suggested to participate in the pathogenesis and development of AP, in view of the reduced NF- $\mathrm{KB}$ p 65 protein levels under conditions of neutrophil depletion.

The AP model was successfully established in C57BL/6 mice with the administration of two doses of L-arginine $(4 \mathrm{~g} / \mathrm{kg}) 1 \mathrm{~h}$ apart. Plasma amylase and MPO activities in pancreas were increased significantly at $48 \mathrm{~h}$ and peaked at $72 \mathrm{~h}$, compared with those in control 


\section{Cellular Physiology Cell Physiol Biochem 2015;35:2111-2120 \begin{tabular}{c|c|c|}
\hline DOI: 10.1159/000374017 & (0) 2015 S. Karger AG, Basel
\end{tabular} and Biochemistry Published online: April 07, 2015 www.karger.com/cpb \\ Chen/Xu/Li/Lu: Role of Neutrophil Depletion in AP Protection}

mice. Histopathological examination revealed tissue damage in pancreas and lung over time after induction of AP, consistent with the time-points at which plasma amylase in pancreas and MPO activities in pancreas and lung increased. Induction of AP additionally resulted in infiltration of leukocytes into pancreas, particularly neutrophils, similarly increasing at $48 \mathrm{~h}$ and peaking at $72 \mathrm{~h}$.

Accumulating evidence supports a crucial role of neutrophils in the progress of AP. Steer and colleagues found that in a choline-deficient ethionine (CDE) diet-induced mouse AP model, neutrophil depletion by pretreatment with antineutrophil serum reduces the severity of AP and completely prevents lung injury [13]. Another study by Regnér and co-workers showed that neutrophil depletion with the anti-Gr-1 antibody reduces the pancreatic trypsinogen activation peptide (TAP) level, plasma amylase and MPO activities in taurocholate-induced severe AP, confirming that neutrophils are critical in mediating pancreatic and lung tissue damage under these conditions [27]. The effects of neutrophil depletion on the L-arginine-induced mouse AP model presented in this study are consistent with previous findings.

Inflammatory factors, such as TNF- $\alpha$ and IL-6, are known to play critical roles in the development of AP [28]. In the present study, TNF- $\alpha$, IL-6, and IL-10 plama levels increased significantly in AP group. Notably, neutrophil depletion led to reduced plasma TNF- $\alpha$ and IL6 , but increased IL-10 levels. Our results suggest a crucial role of neutrophil in the regulation of inflammatory cytokines.

The signaling mechanism by which neutrophil depletion protects against AP is of significant interest. To this end, the pleiotropic transcription factor, NF- $\kappa B$, is known to have a key function in the pathogenesis of AP. Activation of NF- $\kappa B$ is an early event in AP and promotes inflammatory reactions associated with severe pancreatitis [29], suggesting a pro-inflammatory role of NF- $\kappa \mathrm{B}$ in development of the disease [30]. Expression of NF- $\kappa \mathrm{B}$ is correlated with the severity of pancreatitis in a cerulein-induced AP mouse model and its inhibition has a positive role in improving survival of rats in a taurocholate-induced AP model [31, 32]. Among the NF- $\kappa B$ family members, NF- $\kappa B$ p65 plays crucial roles in the progression of AP [33]. In view of these findings, we examined the NF- $\kappa B$ p65 expression patterns. Our experiments showed that NF- $\kappa \mathrm{B}$ p65 is upregulated during L-arginine-induced AP and downregulated upon depletion of neutrophils.

In conclusion, data from our present study clearly demonstrate that neutrophil depletion provides a novel effective therapeutic strategy for the management of L-arginine-induced AP, as evident from the resulting improvement in pancreatic injury, decreased ROS generation and cell apoptosis, and downregulation of NF- $\kappa \mathrm{B}$ in an L-arginine-induced AP mouse model.

\section{References}

1 Frossard JL, Steer ML, Pastor CM: Acute pancreatitis. Lancet 2008;371:143-152.

2 Baron TH, Morgan DE: Acute necrotizing pancreatitis. N Engl J Med 1999;340:1412-1417.

3 Li XY, Wang XB, Liu XF, Li SG: Prevalence and risk factors of organ failure in patients with severe acute pancreatitis. World J Emerg Med 2010;1:201-204.

4 Xiao WQ, Yin GJ, Fan YT, Qiu L, Cang XF, Yu G, Hu YL, Xing M, Wu de Q Wang XP, Hu GY, Wan R: Catalpol ameliorates sodium taurocholate-induced acute pancreatitis in rats via inhibiting activation of nuclear factor kappa b. Int J Mol Sci 2014;15:11957-11972.

5 Awla D, Zetterqvist AV, Abdulla A, Camello C, Berglund LM, Spegel P, Pozo MJ, Camello PJ, Regner S, Gomez MF, Thorlacius H: Nfatc3 regulates trypsinogen activation, neutrophil recruitment, and tissue damage in acute pancreatitis in mice. Gastroenterology 2012;143:1352-1360.

6 Rakonczay Z Jr, Hegyi P, Takacs T, McCarroll J, Saluja AK: The role of nf-kappab activation in the pathogenesis of acute pancreatitis. Gut 2008;57:259-267.

$7 \quad$ Bhatia M: Inflammatory response on the pancreatic acinar cell injury. Scand J Surg 2005;94:97-102.

8 Fortunato F, Burgers H, Bergmann F, Rieger P, Buchler MW, Kroemer G, Werner J: Impaired autolysosome formation correlates with lamp-2 depletion: Role of apoptosis, autophagy, and necrosis in pancreatitis. Gastroenterology 2009;137:350-360. 


\section{Cellular Physiology Cell Physiol Biochem 2015;35:2111-2120 and Biochemistry POI: 10.1159/000374017 $\quad$ (c) 2015 S. Karger AG, Base \\ Chen/Xu/Li/Lu: Role of Neutrophil Depletion in AP Protection}

9 Yu G, Wan R, Yin G, Xiong J, Hu Y, Xing M, Cang X, Fan Y, Xiao W, Qiu L, Wang X, Hu G: Diosmetin ameliorates the severity of cerulein-induced acute pancreatitis in mice by inhibiting the activation of the nuclear factorkappab. Int J Clin Exp Pathol 2014;7:2133-2142.

10 Seyhun E, Malo A, Schafer C, Moskaluk CA, Hoffmann RT, Goke B, Kubisch CH: Tauroursodeoxycholic acid reduces endoplasmic reticulum stress, acinar cell damage, and systemic inflammation in acute pancreatitis. Am J Physiol Gastrointest Liver Physiol 2011;301:G773-782.

11 Chen J, Cai QP, Shen PJ, Yan RL, Wang CM, Yang DJ, Fu HB, Chen XY: Netrin-1 protects against l-arginineinduced acute pancreatitis in mice. PLoS One 2012;7:e46201.

12 Rakonczay Z, Jr., Jarmay K, Kaszaki J, Mandi Y, Duda E, Hegyi P, Boros I, Lonovics J, Takacs T: Nf-kappab activation is detrimental in arginine-induced acute pancreatitis. Free Radic Biol Med 2003;34:696-709.

13 Bhatia M, Saluja AK, Hofbauer B, Lee HS, Frossard JL, Steer ML: The effects of neutrophil depletion on a completely noninvasive model of acute pancreatitis-associated lung injury. Int J Pancreatol 1998;24:77-83.

14 Pastor CM, Vonlaufen A, Georgi F, Hadengue A, Morel P, Frossard JL: Neutrophil depletion--but not prevention of kupffer cell activation--decreases the severity of cerulein-induced acute pancreatitis. World J Gastroenterol 2006;12:1219-1224.

15 Gukovskaya AS, Vaquero E, Zaninovic V, Gorelick FS, Lusis AJ, Brennan ML, Holland S, Pandol SJ: Neutrophils and nadph oxidase mediate intrapancreatic trypsin activation in murine experimental acute pancreatitis. Gastroenterology 2002;122:974-984.

16 Liu ZX, Han D, Gunawan B, Kaplowitz N: Neutrophil depletion protects against murine acetaminophen hepatotoxicity. Hepatology 2006;43:1220-1230.

17 Dawra R, Sharif R, Phillips P, Dudeja V, Dhaulakhandi D, Saluja AK: Development of a new mouse model of acute pancreatitis induced by administration of l-arginine. Am J Physiol Gastrointest Liver Physiol 2007;292:G1009-1018.

18 Zhou X, Xue C: Ghrelin inhibits the development of acute pancreatitis and nuclear factor kappab activation in pancreas and liver. Pancreas 2009;38:752-757.

19 Kruse-Jarres JD, Kaiser C, Hafkenscheid JC, Hohenwallner W, Stein W, Bohner J, Klein G, Poppe W, Rauscher E: Evaluation of a new alpha-amylase assay using 4.6-ethylidene-(g7)-1-4-nitrophenyl-(g1)-alpha-dmaltoheptaoside as substrate. J Clin Chem Clin Biochem 1989;27:103-113.

20 Tang D, Kang R, Livesey KM, Cheh CW, Farkas A, Loughran P, Hoppe G, Bianchi ME, Tracey KJ, Zeh HJ, 3rd, Lotze MT: Endogenous hmgb1 regulates autophagy. J Cell Biol 2010;190:881-892.

21 Bhartiya D, Mundekar A, Mahale V, Patel H: Very small embryonic-like stem cells are involved in regeneration of mouse pancreas post-pancreatectomy. Stem Cell Res Ther 2014;5:106.

22 Pezzilli R, Ceciliato R, Barakat B, Corinaldesi R: Immune-manipulation of the inflammatory response in acute pancreatitis. What can be expected? JOP 2004;5:115-121.

23 Jaeschke H, Smith CW: Mechanisms of neutrophil-induced parenchymal cell injury. J Leukoc Biol 1997;61:647-653.

24 Whitcomb DC: Clinical practice. Acute pancreatitis. N Engl J Med 2006;354:2142-2150.

25 Banks PA, Freeman ML: Practice guidelines in acute pancreatitis. Am J Gastroenterol 2006;101:2379-2400.

26 Sandoval D, Gukovskaya A, Reavey P, Gukovsky S, Sisk A, Braquet P, Pandol SJ, Poucell-Hatton S: The role of neutrophils and platelet-activating factor in mediating experimental pancreatitis. Gastroenterology 1996;111:1081-1091.

27 Abdulla A, Awla D, Thorlacius H, Regner S: Role of neutrophils in the activation of trypsinogen in severe acute pancreatitis. J Leukoc Biol 2011;90:975-982.

28 Pereda J, Sabater L, Aparisi L, Escobar J, Sandoval J, Vina J, Lopez-Rodas G, Sastre J: Interaction between cytokines and oxidative stress in acute pancreatitis. Curr Med Chem 2006;13:2775-2787.

29 Dawra R, Sah RP, Dudeja V, Rishi L, Talukdar R, Garg P, Saluja AK: Intra-acinar trypsinogen activation mediates early stages of pancreatic injury but not inflammation in mice with acute pancreatitis. Gastroenterology 2011;141:2210-2217.

30 Chen X, Ji B, Han B, Ernst SA, Simeone D, Logsdon CD: Nf-kappab activation in pancreas induces pancreatic and systemic inflammatory response. Gastroenterology 2002;122:448-457.

31 Huang H, Liu Y, Daniluk J, Gaiser S, Chu J, Wang H, Li ZS, Logsdon CD, Ji B: Activation of nuclear factorkappab in acinar cells increases the severity of pancreatitis in mice. Gastroenterology 2013;144:202-210.

32 Satoh A, Shimosegawa T, Fujita M, Kimura K, Masamune A, Koizumi M, Toyota T: Inhibition of nuclear factor-kappab activation improves the survival of rats with taurocholate pancreatitis. Gut 1999;44:253258.

33 Treiber M, Neuhofer P, Anetsberger E, Einwachter H, Lesina M, Rickmann M, Liang S, Kehl T, Nakhai H, Schmid RM, Algul H: Myeloid, but not pancreatic, rela/p65 is required for fibrosis in a mouse model of chronic pancreatitis. Gastroenterology 2011;141:1473-1485. 